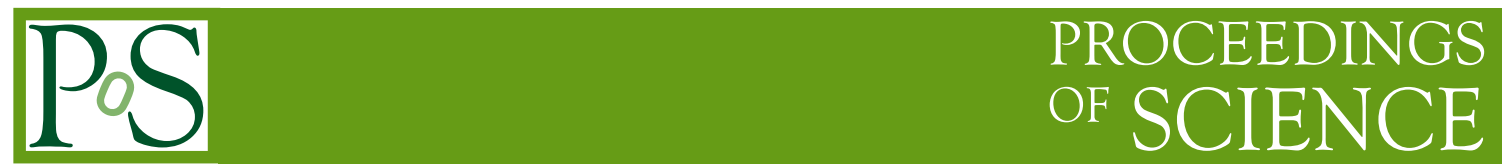

\title{
Top-quark mass determination at the LHC: a theory overview
}

\section{Gennaro Corcella*}

INFN, Laboratori Nazionali di Frascati

Via E. Fermi 40, 00044 Frascati (RM), Italy

E-mail: gennaro.corcella@lnf.infn.it

I briefly overview the methods employed at the LHC to extract the top-quark mass, taking particular care about the theory uncertainty and the dependence on the Monte Carlo hadronization parameters.

The European Physical Society Conference on High Energy Physics

5-12 July, 2017

Venice

* Speaker. 


\section{Introduction}

The mass of the top quark is a fundamental parameter of the Standard Model, since it enters in the electroweak precision tests [1] and constrained the mass of the Higgs boson even before its actual discovery at the LHC. Moreover, the fact that the electroweak vacuum lies on the boundary between stability and metastability regimes [2] depends on the actual values of top and Higgs masses. This statement does however depend on the identification of the top-quark mass world average, i.e. $m_{t}=[173.34 \pm 0.27$ (stat) \pm 0.71 (syst) $] \mathrm{GeV}$ [3], with the pole mass and no extra uncertainty is included in the exploration of Ref. [2]. In fact, any change of the central value or of the error on $m_{t}$ may affect the results in [2], to the point of even moving the vacuum position inside the stability or instability regions. It is therefore of paramount importance determining $m_{t}$ at the LHC with the highest possible precision and, above all, estimating reliably all sources of uncertainty.

The top-quark mass is determined by comparing experimental data with theory predictions: the extracted mass is the quantity $m_{t}$ in the calculation or in the Monte Carlo event generator employed to simulate top production and decay. In the following, I shall review the main methods used to reconstruct the top-quark mass at the LHC and discuss the theoretical and Monte Carlo uncertainties, paying special attention to the dependence on the event-generator $b$-fragmentation parameters. I shall finally make some concluding remarks.

\section{Top-quark mass extraction at LHC}

Top-quark mass determinations at hadron colliders are classified as standard or alternative measurements. Standard top-mass analyses adopt the template, matrix-element and ideogram methods (see, e.g., the analyses in $[4,5]$ ) and compare final-state distributions, associated with topdecay $(t \rightarrow b W)$ products, such as the $b$-jet+lepton invariant mass in the dilepton channel, with the predictions yielded by the Monte Carlo codes. Event generators like the general-purpose HERWIG [6] or PYTHIA [7] simulate the hard-scattering process at leading order (LO), multi-parton emissions in the soft or collinear approximation and the interference between top-production and decay stages is neglected (narrow-width approximation). More recent NLO+shower programs, such as MadGraph5_aMC@NLO [8] and POWHEG [9], implement NLO hard-scattering amplitudes, but still depend on HERWIG and PYTHIA for parton cascades and non-perturbative phenomena, such as hadronization or underlying event. As a whole, standard top-quark mass determinations, as they are based on the reconstruction of the invariant mass of the top-decay products and rely on programs which factorize top production ad decay, should lead to results close to the top-quark pole mass. However, as will be pointed out hereafter, a careful determination of the theoretical uncertainty, of both perturbative and non-perturbative origins, such as missing higher orders, width corrections and colour-reconnection effects, is compelling.

Other strategies to measure $m_{t}$, making use of total or differential cross sections, endpoints, energy peaks or kinematic properties of $t \bar{t}$ final states, are traditionally called 'alternative' measurements. The total $t \bar{t}$ cross section was calculated in the NNLO+NNLL approximation [12] and allows a direct determination of the pole mass $[10,11]$, the mass definition used in the computation [12]. The errors in [10] and [11] are larger than those in the standard methods; however, they 
are expected to decrease thanks to the higher statistics foreseen at the LHC Run II. Moreover, the dependence on the mass implemented in the Monte Carlo program, employed to obtain the acceptance, in very mild. The top pole mass was also extracted from the measurement of the $t \bar{t}+1$ jet cross section, more sensitive to $m_{t}$ than the inclusive $t \bar{t}$ rate [13, 14]. In Ref. [15], the NLO $t \bar{t} j$ cross section was calculated through the POWHEG-BOX, using the pole mass, and matched to PYTHIA. Reference [16] computed instead the NLO $t \bar{t} j$ rate in terms of the $\overline{\mathrm{MS}}$ mass and compared the result with the LHC measurements: the values of pole and $\overline{\mathrm{MS}}$ masses, extracted by following the methods in [15] and [16], are nonetheless in agreement.

Other proposed methods to reconstruct $m_{t}$ rely on kinematic properties of top-decay final states. It was found that the peak of the energy of the $b$-jet in top decay at LO is independent of the boost from the top to the laboratory frame, as well as of the production mechanism [17]. The CMS Collaboration measured the top mass from the $b$-jet energy peak data at $8 \mathrm{TeV}$ in [18]. The $b$-jet+lepton invariant-mass $\left(m_{b \ell}\right)$ spectrum was used by CMS to reconstruct $m_{t}$ in the dilepton channel, by comparing the data with PYTHIA [19]. The endpoints of distributions like $m_{b \ell}, \mu_{b b}$ and $\mu_{\ell \ell}$, where $\mu_{b b}$ and $\mu_{\ell \ell}$ are a generalization of the $b \bar{b}$ and $\ell^{+} \ell^{-}$invariant masses in the dilepton channel, $b$ being a $b$-jet in top decay, were also explored to constrain $m_{t}$ [20]. Since $b$-flavoured jets can be calibrated directly from data, Monte Carlo uncertainties in the endpoints are mostly due to colour reconnection.

Finally, purely leptonic observables in the dilepton channel, such as the Mellin moments of lepton energies or transverse momenta, were proposed to measure $m_{t}$ as they do not require the reconstruction of the top quarks [21]. Such quantities exhibit pretty small hadronization effects, but they are sensitive to the production mechanism, to the Lorentz boost from the top rest frame to the laboratory frame, as well as to higher-order corrections. Preliminary analyses have been carried out in [22] (CMS, based on LO MadGraph) and [23] (ATLAS, based on the MCFM NLO parton-level code [24]) and are expected to be improved by matching NLO amplitudes with shower/hadronization generators.

\section{Theory and Monte Carlo uncertainties in the top-mass extraction}

In Ref. [3], where the extraction of the world average is described, the theory uncertainty accounts for about $540 \mathrm{MeV}$ of the overall $710 \mathrm{GeV}$ systematics. In particular, Ref. [3] distinguishes the contributions due to Monte Carlo generators, radiation effects, colour reconnection and parton distribution functions (PDFs).

The Monte Carlo systematics is due to the differences in the implementation of parton showers, matrix-element matching, hadronization and underlying event in the various programs available to describe top-quark production and decay. There is no unique way to estimate this uncertainty, though: one can either compare two different generators or choose a code and explore how its predictions fare with respect to variations of the parameters. For example, in [3], CDF compares HERWIG and PYTHIA, while D0 uses ALPGEN+PYTHIA and ALPGEN+HERWIG [25]; both Tevatron experiments use MC@NLO to gauge the overall impact of NLO corrections. At the LHC, ATLAS compares MC@NLO with POWHEG for the NLO contributions and PYTHIA with HERWIG for shower and hadronization; CMS instead confronts MadGraph with POWHEG. 


\begin{tabular}{|c|c|c|c|c|c|c|c|c|c|c|}
\hline \multirow{2}{*}{0} & \multirow[b]{2}{*}{$\Delta_{O}^{m}$} & \multicolumn{9}{|c|}{$\Delta_{\theta}^{m}$} \\
\hline & & PSPLT(2) & QCDLAM & CLPOW & CLSMR(2) & CLMAX & RMASS(5) & RMASS(13) & $\begin{array}{l}\text { VGCUT } \\
\end{array}$ & $\begin{array}{l}\text { VQCUT } \\
\end{array}$ \\
\hline$m_{B \ell}$ & 0.52 & $0.036(4)$ & $\begin{array}{l}-0.008(2) \\
\end{array}$ & $-0.007(5)$ & $0.002(3)$ & $-0.007(4)$ & $0.058(1)$ & $0.06(5)$ & $0.003(1)$ & $-0.003(3)$ \\
\hline $\begin{array}{l}p_{T, B} \\
p_{T, B}\end{array}$ & 0.47 & $0.072(1)$ & $-0.03(9)$ & $-0.02(7)$ & $0.0035(5)$ & $-0.03(5)$ & $0.11(9)$ & $0.12(5)$ & $0.0066(2)$ & $-0.006(5)$ \\
\hline$E_{B}$ & 0.43 & $0.069(7)$ & & -0.0 & & & & $0.12(2)$ & $0.006(2)$ & $-0.007(5)$ \\
\hline$E_{B}$ & 0.13 & $0.0005(5)$ & $-0.04(3)$ & $0.04(2)$ & $-0.0002(2)$ & $-0.004(4)$ & $0.008(3)$ & $0.008(2)$ & $\begin{array}{l}-0.002(5) \\
-0.05\end{array}$ & $0.008(2)$ \\
\hline
\end{tabular}

Table 1: Dependence of $m_{t}$ HERWIG 6 shower and hadronization parameters.

The radiation uncertainty gauges the effect of initial- and final-state radiation on the top mass and is typically obtained by varying in suitable ranges the relevant parameters in the parton-shower generators. Concerning PDFs, there are strategies to gauge the induced error on $m_{t}$ in the different experiments, although using two different sets or a given set but with different parametrizations are common trends.

Colour reconnection is another source of error on $m_{t}$, accounting for about $310 \mathrm{MeV}$ in [3]: the very fact that, for example, a bottom quark in top decay $(t \rightarrow b W)$ can be colour-connected to an initial-state antiquark does not have its counterpart in $e^{+} e^{-}$annihilation and therefore its modelling in Monte Carlo event generators may need retuning at hadron colliders. Moreover, this phenomenon is an irreducible uncertainty in the interpretation of the measured mass as a pole mass. Investigations on the impact of colour reconnection on $m_{t}$ were undertaken in [26, 27], in the frameworks of PYTHIA and HERWIG, respectively. In particular, Ref. [27] addresses this issue by simulating fictitious top-flavoured hadrons in HERWIG and comparing final-state distributions, such as the $B W$ invariant mass, with standard $t \bar{t}$ events. In fact, in the top-hadron case, assuming $T$ decays according to the spectator model, the $b$ quark is forced to connect with the spectator or with antiquarks in its own shower, namely $b \rightarrow b g$, followed by $g \rightarrow q \bar{q}$, and colour reconnection is suppressed. Furthermore, the analysis [27] may also serve to address the relation between the measured mass, often called 'Monte Carlo' mass, with the pole mass, since the mass of a $T$ hadron can be related to any top-quark mass definition by means of lattice, potential models or Non Relativistic QCD.

More recently, work has been carried out to assess the dependence of the top-quark mass, extracted by means of the Mellin moments $\mathscr{M}_{n}$ of some variables related to $B$-hadrons in top decays, on the Monte Carlo shower and hadronization parameters [28], extending the investigation in [29], which studied only the $m_{B \ell}$ quantity. In fact, when addressing $B$-hadron rather than $b$-jet observables, one should deal with fragmentation uncertainties, rather than with the jet-energy scale, entering in measurements relying on $b$-jets. If $\mathscr{M}_{1}=\langle O\rangle$ is the average value of some observable $O$ and $\theta$ a generic generator parameter, one can write the following relations:

$$
\frac{d m_{t}}{m_{t}}=\Delta_{O}^{m} \frac{d\langle O\rangle}{\langle O\rangle} ; \frac{d\langle O\rangle}{\langle O\rangle}=\Delta_{\theta}^{O} \frac{d \theta}{\theta} \Rightarrow \frac{d m_{t}}{m_{t}}=\Delta_{\theta}^{m} \frac{d \theta}{\theta},
$$

where we defined $\Delta_{\theta}^{m}=\Delta_{O}^{m} \Delta_{\theta}^{O}$. Therefore, if one requires, e.g., a relative error below $0.3 \%$ on $m_{t}$, namely $d m_{t} / d m_{t}<0.003$, one should also have $\Delta_{\theta}^{m}(d \theta / \theta)<0.003$. In Table 1 , we present the $\Delta$ factors $\Delta_{O}^{m}$ and $\Delta_{\theta}^{m}$, assuming that the top mass is extracted in the dilepton channel by means of the first Mellin moment of observables $O$, like the $B \ell$ invariant mass, the energy $E_{B}$ and the transverse momentum $p_{T, B}$ of $B$ hadrons in top decays, the energy $E_{\ell}$ of charged leptons in $W$ decays. The $\theta$ entries in Table 1 are parameters of the HERWIG 6 event generator, implementing the cluster hadronization model. In detail, PSPLT(2) is a parameter ruling the mass distribution of 
the decays of $b$-flavoured clusters, while CLMAX(2) and CLPOW determine the highest allowed cluster mass. Furthermore, unlike Ref. [29], which just accounted for cluster-hadronization parameters, Ref. [28] also investigates the dependence of top-quark mass observables on the following parameters: RMASS(5) and RMASS(13), the bottom and gluon effective masses, respectively, and the virtuality cutoffs, VQCUT for quarks and VGCUT for gluons, which are added to the parton masses in the shower. The impact of changing QCDLAM, the HERWIG parameter playing the role of an effective $\Lambda_{\mathrm{QCD}}$ in the shower definition of the strong coupling constant [30], is also examined. Overall, from Table 1 one learns that, if one aims at $d m_{t} / m_{t}<0.003$, the parameters PSPLT(2), QCDLAM, CLPOW, CLMAX and the $b$-quark and gluon effective masses are to be known with a relative precision of $10 \%$. The dependence of $m_{t}$ on CLSMR(2) and the cutoffs VQCUT and VGCUT is instead very mild and, in principle, it would be sufficient determining only the order of magnitude of such parameters to meet a $0.3 \%$ goal on $m_{t}$. More details on the dependence of the top-quark mass on hadronization and shower parameters will be soon available in [28].

Another recent investigation on the sensitivity of $m_{t}$ to the Monte Carlo modelling was carried out in Ref. [31], where the authors investigated the PYTHIA uncertainty in $m_{t}$ in the lepton+jets channel. It was then found that the error on the top mass can be significantly reduced if one calibrates the $W$ mass or applies the soft-drop jet grooming.

On the top of the uncertainties in the $m_{t}$ determination at the LHC, there are long-standing theoretical issues affecting the accuracy on the top mass, namely the interpretation of the measured quantity in terms of the pole mass and the renormalon ambiguity on the pole mass: a review on such topics can be found in [32]. In fact, Ref. [33] compared PYTHIA with a NLO+NNLL SCET calculation for $e^{+} e^{-} \rightarrow t \bar{t}$ annihilation and calibrated the top mass used in the computation, the so-called MSR definition, to agree with the Monte Carlo 2-jettiness distribution. Within the error range, the mass parameter in PYTHIA is consistent with the fitted value of $m_{\mathrm{MSR}}(1 \mathrm{GeV})$, while $(0.57 \pm 0.28) \mathrm{GeV}$ is the discrepancy with respect to the corresponding pole mass. Ref. [34] proposed instead the measurement of $m_{t}$ in $p p$ collisions by using boosted top jets with light softdrop grooming, in lepton+jets and all-jet final states. The groomed top-jet mass spectrum was then calculated resumming soft- and collinear-enhanced contributions in the NLL approximation and compared with the spectra yielded by PYTHIA, trying to calibrate the PYTHIA mass parameter to reproduce the resummed distribution. The result of this calibration is that the pole mass is about 400-700 MeV smaller than the tuned mass in the Monte Carlo generator.

As for the renormalon ambiguity, two recent analyses, i.e. Refs. [35] and [36], estimated the uncertainty in the top pole mass due to renormalons, obtaining about 110 and $250 \mathrm{MeV}$, respectively: though differing by about a factor of 2 , such results are both smaller than the current error on the measured top mass.

\section{Conclusions}

I discussed the main strategies to determine the top mass at the LHC, emphasizing the role played by the theory uncertainties. The so-called standard measurements, such as those relying on template, matrix-element or ideogram methods, are based on the reconstruction of the top-decay products, and therefore they yield results close to the top-quark pole mass: however, a careful exploration of the theory error is mandatory. In particular, the ongoing work in [27], studying 
fictitious top-flavoured hadrons, should shade light on both colour reconnection and relation between measured mass and pole mass. It will be therefore very interesting to compare the eventual uncertainties according to the method in [27] with the errors obtained in [33, 34], comparing resummations and event generators, as well as with the renormalon ambiguity gauged in $[35,36]$.

Among the alternative measurements, extracting $m_{t}$ by confronting the $t \bar{t}$ and $t \bar{t} j$ cross sections with NLO or NNLO calculations allows a clean extraction of the pole mass. The errors are substantially larger than in the standard measurements, but nonetheless they are supposed to become much smaller once the LHC statistics increase. Other methods, employing endpoints, leptonic observables or observables like the $b$-jet+lepton mass distribution, are very interesting and worthwhile to be further developed, since they are sensitive to different effects with respect to the standard determination. For example, the endpoint method minimizes the impact of the Monte Carlo generators, while leptonic quantities do not need the reconstruction of the top quarks.

Particular care was taken in this talk about the Monte Carlo uncertainty in the $m_{t}$ determination, namely the dependence of $m_{t}$ on hadronization parameters, once it is measured from $B$-hadron observables, such as the $B \ell$ invariant mass or the $B$-energy or transverse-momentum spectra. I presented some results yielded by the HERWIG event generator, showing that most parameters are to be tuned with an accuracy of at least $10 \%$, for the sake of meeting a $0.3 \%$ precision goal on $m_{t}$. More details on this investigation and on the dependence of $m_{t}$ on the parameters of PYTHIA, the other multi-purpose parton shower generator employed in the analysis, will be soon available in [28]. It will be challenging comparing the hadronization uncertainties obtained in [28] with those relying on NLO+shower generators, such as the recent $t \bar{t}$ POWHEG implementation presented in [37], accounting for non-resonant contributions and for the interference between top production and decay. Furthermore, at parton-level, the full process $p p \rightarrow W^{+} W^{-} b \bar{b} \rightarrow\left(\ell^{+} v_{\ell}\right)\left(\ell^{-} \bar{v}_{\ell}\right)$ was lately computed at NLO and compared with scenarios where NLO $t \bar{t}$ production is matched with different top-decay modelling, namely LO and NLO top decays in the narrow-width approximation, as well as parton showers [38]. It will be certainly very interesting confronting the approaches in Refs. [37] and [38], and the induced error in the $m_{t}$ determination.

In summary, the current world-average $m_{t}$ analysis exhibits an uncertainty of about $0.5 \%$ and higher accuracies are foreseen in the near future, thanks to the large statistics. Given the relevance of $m_{t}$ in the Standard Model, any progress in improving the present higher-order calculations and Monte Carlo event generators, for the sake of assessing reliably the theoretical error, including the interpretation of the measurements in terms of the pole mass, will therefore be especially desirable.

\section{References}

[1] Gfitter Group, Eur. Phys. J. C74 (2014) 3046.

[2] G. Degrassi, S. Di Vita, J. Elias-Miró, J.R. Espinosa, G.F. Giudice, G. Isidori and A. Strumia, JHEP 1208 (2012) 098.

[3] ATLAS and CDF and CMS and D0 Collaborations, arXiv:1403.4427 [hep-ex].

[4] ATLAS Collaboration, Phys. Lett. B761 (2016) 350.

[5] CMS Collaboration, Phys. Rev. D93 (2016) 072004.

[6] J. Bellm et al., Eur. Phys. J. C76 (2016) 196. 
[7] T. Sjöstrand et al., Comput. Phys. Commun. 191 (2015) 159.

[8] J. Alwall et al., JHEP 1407 (2014) 079.

[9] S. Alioli, P. Nason, C. Oleari and E. Re, JHEP 1006 (2010) 043.

[10] ATLAS Collaboration, Eur. Phys. J. C74 (2015) 3109.

[11] CMS Collaboration, JHEP 08 (2016) 029.

[12] M. Czakon, P. Fiedler and A. Mitov, Phys. Rev. Lett. 110 (2013) 252004.

[13] ATLAS Collaboration, JHEP 1510 (2015) 121.

[14] CMS Collaboration, CMS-PAS-TOP-13-006.

[15] S. Alioli, P. Fernandez, J. Fuster, A. Irles, S.-O. Moch, P. Uwer and M. Vos, Eur. Phys. J. C73 (2013) 2438.

[16] J. Fuster, D. Melini, P. Uwer and M. Vos, arXiv:1704.00540 [hep-ph].

[17] K. Agashe, R. Franceschini, D. Kim and M. Schulze, Eur. Phys. J. C76 (2016) 636.

[18] CMS Collaboration, PoS ICHEP2016 (2016) 743.

[19] CMS Collaboration, CMS-PAS-TOP-14-014.

[20] CMS Collaboration, Eur. Phys. J. C73 (2013) 2494.

[21] S. Frixione and A. Mitov, JHEP 1409 (2014) 012.

[22] CMS Collaboration, CMS-PAS-TOP-16-002.

[23] ATLAS Collaboration, ATLAS-CONF-2017-044.

[24] J.M. Campbell and R.K. Ellis, J. Phys. G42 (2015) 015005.

[25] M.L. Mangano, M. Moretti, F. Piccinini, R. Pittau, A. Polosa, JHEP 0307 (2003) 001.

[26] S. Argyropoulos and T. Sjöstrand, JHEP 1411 (2014) 043.

[27] G. Corcella, EPJ Web Conf. 80 (2014) 00019.

[28] G. Corcella, R. Franceschini and D. Kim, in preparation.

[29] G. Corcella and F. Mescia, Eur. Phys. J. C65 (2010) 171; Erratum-ibid. C68 (2010) 687.

[30] S. Catani, B.R. Webber, and G. Marchesini, Nucl. Phys. B349 (1991) 635.

[31] A. Andreassen and M.D. Schwartz, JHEP 1710 (2017) 151.

[32] G. Corcella, arXiv:1709.09878 [hep-ph].

[33] M. Butenschön, B. Denhadi, A.H. Hoang, V. Mateu, M. Preisser and I.W. Stewart, Phys. Rev. Lett. 117 (2016) 232001.

[34] A.H. Hoang, S. Mantry, A. Pathak and I.W. Stewart, arXiv:1708.02586 [hep-ph].

[35] M. Beneke, P. Marquard, P. Nason and M. Steinhauser, Phys. Lett. B775 (2017) 63.

[36] A.H. Hoang, C. Lepenik and M. Preisser, JHEP 1709 (2017) 099.

[37] T. Jezo, J.M. Lindert, P. Nason, C. Oleari and S. Pozzorini, Eur. Phys. J. C76 (2016) 691.

[38] G. Heinrich, A. Maier, R. Nisius, J. Schlenk, M. Schulze, L. Scyboz and J. Winter, arXiv:1709.08615 [hep-ph]. 